\title{
Nanoscale magnetization reversal by electric field-induced ion migration
}

\begin{abstract}
Qilai Chen, CAS Key Laboratory of Magnetic Materials and Devices, Ningbo Institute of Materials Technology and Engineering, Chinese Academy of Sciences, Ningbo, Zhejiang 315201, China; School of Mechanical Engineering, Xiangtan University, Xiangtan, Hunan 411105, China; Zhejiang Province Key Laboratory of Magnetic Materials and Application Technology, Ningbo Institute of Materials Technology and Engineering, Chinese Academy of Sciences, Ningbo 315201, China

Gang Liu, Shuang Gao, Xiaohui Yi, and Wuhong Xue, CAS Key Laboratory of Magnetic Materials and Devices, Ningbo Institute of Materials Technology and Engineering, Chinese Academy of Sciences, Ningbo, Zhejiang 315201, China; Zhejiang Province Key Laboratory of Magnetic Materials and Application Technology, Ningbo Institute of Materials Technology and Engineering, Chinese Academy of Sciences, Ningbo 315201, China

Minghua Tang, School of Materials Science and Engineering, Xiangtan University, Xiangtan, Hunan 411105, China

Xuejun Zheng, School of Mechanical Engineering, Xiangtan University, Xiangtan, Hunan 411105, China

Run-Wei Li, CAS Key Laboratory of Magnetic Materials and Devices, Ningbo Institute of Materials Technology and Engineering, Chinese Academy of Sciences, Ningbo, Zhejiang 315201, China; Zhejiang Province Key Laboratory of Magnetic Materials and Application Technology, Ningbo Institute of Materials Technology and Engineering, Chinese Academy of Sciences, Ningbo 315201, China
\end{abstract}

Address all correspondence to Gang Liu, Xuejun Zheng, Run-Wei Li at liug@nimte.ac.cn, zhengxuejun@xtu.edu.cn, runweili@nimte.ac.cn

(Received 30 May 2018; accepted 29 August 2018)

\begin{abstract}
Nanoscale magnetization modulation by electric field enables the construction of low-power spintronic devices for information storage applications and, etc. Electric field-induced ion migration can introduce desired changes in the material's stoichiometry, defect profile, and lattice structure, which in turn provides a versatile and convenient means to modify the materials' chemical-physical properties at the nanoscale and in situ. In this review, we provide a brief overview on the recent study on nanoscale magnetization modulation driven by electric field-induced migration of ionic species either within the switching material or from external sources. The formation of magnetic conductive filaments that exhibit magnetoresistance behaviors in resistive switching memory via foreign metal ion migration and redox activities is also discussed. Combining the magnetoresistance and quantized conductance switching of the magnetic nanopoint contact structure may provide a future high-performance device for non-von Neumann computing architectures.
\end{abstract}

\section{Introduction}

Spintronic devices, which are enabled by controlling the nanoscale magnetization at room temperature through electric means, have been widely used in the semiconductor industry for the physical implementation of magnetic random access memory, spin-polarized field effect transistors, spin emitting diodes, and, etc. ${ }^{[1-3]}$ Generally, the electric modulation of magnetization reversal is realized by currents and suffers from practical problems that hinder the direct device application. For example, the spin-torque effect that uses the spin-polarized electric current to switch the magnetization of the magnetic domains usually faces the problem of high power consumption, ${ }^{[4]}$ while multiferroic effects that regulate the magnetization through interfacial strain/charges and exchange bias based ferroelectric/ferromagnetic coupling of a composite materials require the presence of external magnetic fields. ${ }^{[5-10]}$ Electrical modulation via carrier concentration and orbital occupation of $3 \mathrm{~d}$ electrons manipulation also needs extra requirements of low working temperature and/or complex sample structures. ${ }^{[1-13]}$ With these concerns, electric field-induced nanoscale magnetization reversal with low power and high-efficiency features is still a challenging work at the moment for spin-based memory and logic systems.

Fortunately, nanoscale ion migration stimulated by external electric field provides alternative possibilities for introducing the desired modulation in materials' stoichiometries, defect profiles and lattice structures, which naturally offers a versatile and convenient platform to fine-tune their chemical and physical properties. ${ }^{[14,15]}$ In particular, the ion-based resistive switching devices with the metal/insulator/metal sandwich structure demonstrate both the adjustable electrical resistance for information storage applications, ${ }^{[16-18]}$ and interesting optical and magnetic phenomena. ${ }^{[19-22]}$ Recently, it is demonstrated that the electric field-induced migration and redistribution of cations can modulate the magnetic anisotropy and magnetization direction of $\mathrm{CoFe}_{2} \mathrm{O}_{4}$ and $\mathrm{LiFe}_{5} \mathrm{O}_{8}$ thin films significantly, presenting an initial yet promising example of ion migration controlled magnetism for the construction of multibit low-power memory devices. ${ }^{[23,24]}$ Local rearrangement and long-range intercalation of ionic species also influence the Curie temperature $\left(T_{\mathrm{C}}\right)$, coercive field $\left(H_{\mathrm{C}}\right)$, and saturated magnetic moment $\left(M_{\mathrm{S}}\right)$ of the magnetic thin films. ${ }^{[21,22,25-27]}$ In 
addition to modulating the magnetization through electrical field-induced ion migration and redistribution, the formation of foreign magnetic conductive filament via the ionization and injection of $\mathrm{Co}^{3+}$ and $\mathrm{Ni}^{2+}$ ions from electrochemically active ferromagnetic metal electrodes can also generate tunneling magnetoresistance (TMR) and anisotropic magnetoresistance (AMR) phenomena in different resistance states. ${ }^{\text {[28-31] }}$ Enrichment of the bistable resistive switching characteristics of memory devices with MR behaviors allows the integration of other functions e.g., sensing and data processing into a single cell, and benefits the future breakthrough of von Neumann bottleneck issue that is faced by the modern computer systems.

In this review paper, we aim to provide a brief overview of the recent study on nanoscale magnetization reversal driven by electric field-induced ion migration. Concentration will be focused primarily discussing on the modulation of magnetization through electric field-induced unidirectional magnetic anisotropy in ferrite nanofilms. The overall effects of ion migration and localized redistribution along a different crystalline direction, and the influence of different migrating cationic species, as assessed experimentally by in-situ scanning probe microscope (SPM) and theoretically with first-principle calculations, are summarized. Modulation of the Curie temperature, the coercive field and the magnetic moment by redistribution of the ionic species within the magnetic structure is further discussed. Following this, the modulation of the magnetism of the ferromagnetic nanofilms by redistribution of ions from the external sources is reviewed. Finally, we discuss the construction of magnetic conductive filaments that exhibit magnetoresistance behaviors in resistive switching memory via foreign metal ion migration and redox activities, and conclude the review with future perspective that anomalous giant magnetoresistance effect may be observed in magnetic nanopoint contact (NPC) structures with quantized conductance and can be utilized for achieving higher-logic computing architectures.

\section{Nanoscale magnetization reversal of ferrite nanofilms induced by local ion migration and redistribution}

In comparison with the (spin-polarized) current-based tuning of magnetism in the conventional energy-consuming spintronic devices, nanoscale magnetization reversal induced by electric field may help offer an alternative energy-efficient way via suppressing the current flowing across the sample. As demonstrated in the resistive switching devices with the structure of metal/insulator/metal, electric field-induced ion migration approach provides such a possibility through modifying the materials' defect profile, chemical composition, and physical properties. Generally, ion migration occurs at local defects such as grain boundaries and, etc., in resistive switching materials, as is directly visualized by monitoring the local conductance of the insulator with conductive atomic force microscope (C-AFM). ${ }^{[32]}$ Transporting through the dangling bonds of the grain boundaries (with relatively large free space), changes in the crystalline structure of the grains and thus strain effects are usually ignored. On the other hand, the migration of mobile ions such as $\mathrm{Li}^{+}$is also observed inside the crystalline grains. Once the migration of mobile ions and the subsequent redox reaction leads to the formation of conductive filaments that connect both the anode and cathodes, the two electrodes become short-circuited and the external electric field is no longer falling onto the insulator. As such, further ion migration ceases and the accumulated ion appears "saturated". More information about the mechanism of ion migration can be referred to in the literature. ${ }^{[15,33,34]}$

In 2015, Chen et al. investigated nanoscale magnetization reversal through electric field induced local ion migration and redistribution in cobalt ferrite (CFO) thin films via SPM technique for the first time. ${ }^{[23]}$ The use of C-AFM and magnetic force microscope (MFM) modes allowed them to monitor the dynamic evolution of the conductance and out-of-plane magnetization of CFO, wherein the correlation between them can offer important insights into the underlying mechanism of ionbased magnetization reversal. (110)-textured CFO thin film is prepared by pulse laser deposition (PLD) technique on $\mathrm{SrRuO}_{3} / \mathrm{SrTiO}_{3}$ (SRO/STO) substrates, and confirmed by X-ray diffractive pattern with a cubic $F m 3 m$ inverse spinel structure. Due to the non-uniform distribution of the ionic species in the plasma glow or the scattering of the ions by the residue gas molecules during PLD preparation, vacancies exist in the as-prepared CFO thin film, which is iron-deficient with the $\mathrm{Fe} / \mathrm{Co}$ atomic ratio of 1.9 as evidenced by the energy-dispersive $\mathrm{x}$-ray spectrum. As shown in Fig. 1, the magnetic domains with the out-of-plane magnetization point up (in yellow) and down (in blue) are distributed randomly whereas showing almost equal total areas [left panels of Figs. 1(c) and 1(e), respectively]. The application of $-3 \mathrm{~V}$ voltage to the sample can tune the magnetization direction of most of the domains to pointing upwards [right panel of Fig. 1(c)], while the application of $3 \mathrm{~V}$ voltage shows an opposite influence [right panel of Fig. 1(e)]. Upon reversing the magnetization of the $\mathrm{Co} / \mathrm{Cr}$ coated tip, a mirror image of the right panel of Figs. 1(c) and 1(e) are obtained, respectively. This confirms that the observed changes in the MFM images under the applied electric field are arising from the magnetic rather than electrostatic interactions between the MFM tip and the sample, and preliminarily suggests that nanoscale electric field-induced magnetization inversion can be realized in the CFO thin film at room temperature. Application of external voltages is applied in the sequence of $0 \mathrm{~V} \rightarrow-4 \mathrm{~V} \rightarrow 0 \mathrm{~V} \rightarrow 4 \mathrm{~V} \rightarrow 0 \mathrm{~V} \rightarrow-4 \mathrm{~V}$ reveals that the electric field induced magnetization modulation is completely reversible [Fig. 2(a)]. By defining the contrast change ratio as that between the total areas of the electrically produced upward-magnetized domains and the initially upward-magnetized domains, $\sim 70 \%$ modulation of the magnetization in CFO thin films is demonstrated [Fig. 2(b)].

For better understanding the underlying mechanism of the observed magnetization reversal in CFO thin films, firstprinciples calculations are performed to verify the relationship between the electric field-induced ion migration and the 
(a)
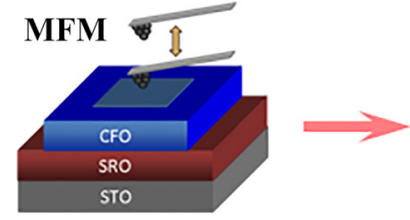

\section{Morphology}
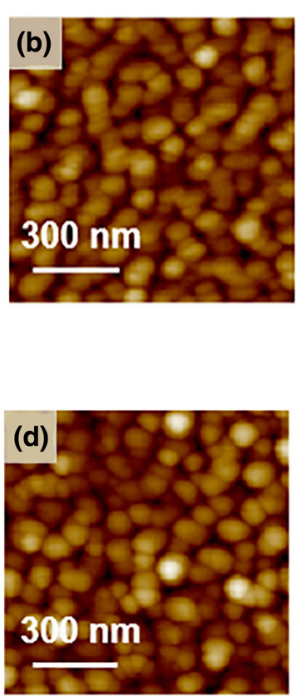

$30 \mathrm{~nm}$
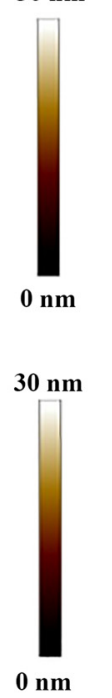
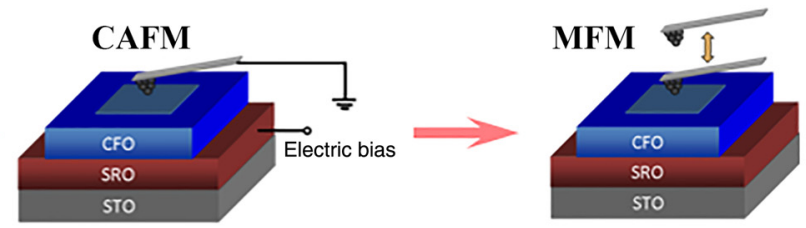

MFM images
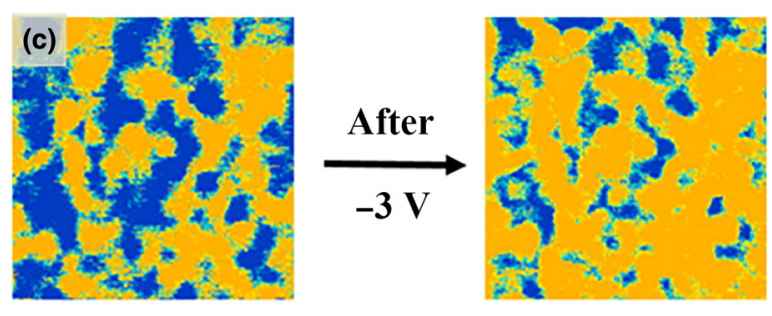

$0.2^{\circ}$
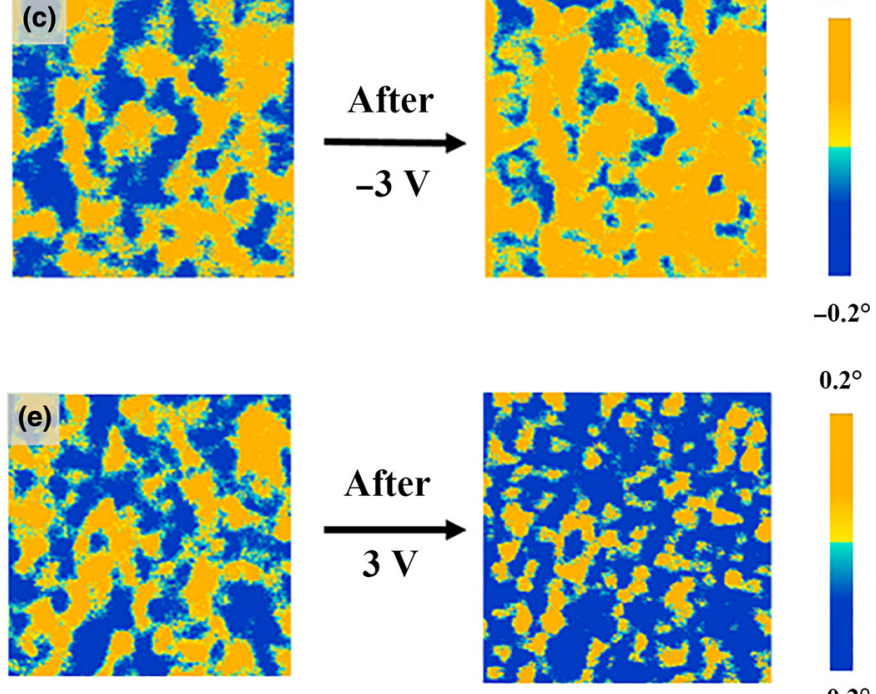

$0.2^{\circ}$

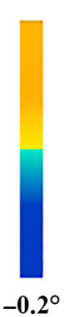

Figure 1. Electric field-induced magnetization reversal in CFO thin films. (a) Schematic illustration of the experimental setups for the C-AFM and MFM measurements. (b), (d) Morphologies of the CFO thin film at the pristine state and (c), (e) corresponding MFM images of the CF0 thin film at the pristine state and after being subjected to biased voltages of -3 and $3 \mathrm{~V}$, respectively. Scale bar: $300 \mathrm{~nm}$. Adapted with permission from Ref. 23 (American Chemical Society, 2015).

magnetic anisotropy of the CFO unit. As shown in Fig. 3(a), A site of the unit cell is occupied by iron species while the B-site-paired $\mathrm{Co}^{2+}$ and $\mathrm{Fe}^{3+}$ ions are alternatively arranged along the $<110\rangle$ direction in an ideal $\mathrm{CoFe}_{2} \mathrm{O}_{4}$. Calculation results suggest that the formation energy of the $\mathrm{B}$-site Fe vacancies is lower than that of the B-site Co vacancies and the A-site $\mathrm{Fe}$ vacancies [Fig. 3(b)]. Therefore, B-site Fe vacancies can be more easily formed in the CFO thin films and are consistent with the experimental $\mathrm{Fe} / \mathrm{Co}$ ratio of 1.9. In the subsequent calculation works, one Fe vacancy is introduced into the $\mathrm{CFO}$ unit cell, while the $\mathrm{CFO}$ thin film with different $\mathrm{Fe} / \mathrm{Co}$ ratio will exhibit different magnetic and magnetization modulation behavior. Changing the composition of the CFO thin films can be realized by modifying the deposition condition, e.g., the composition of the PLD target, sputtering angle, chamber pressure, and, etc. It is noteworthy that the B-site Fe vacancy prefers to stay between two $\mathrm{Co}^{2+}$ ions to lower the total energy of the unit cell [Fig. 3(c)]. When an electric field along the [11 0] direction is applied onto the model system, the neighboring $\mathrm{Co}^{2+}$ ion on the right of the $\mathrm{Fe}$ vacancy will exchange its occupation with the $\mathrm{Fe}$ vacancy, forming a new configuration of $\mathrm{Fe}^{3+}-\mathrm{Co}^{2+}-\mathrm{Co}^{2+}-\mathrm{V}_{\mathrm{FeB}}$ and a lower total energy of the unit cell with its magnetization pointing along the [110] direction [Fig. 3(d)]. With the shift of the uniaxial magnetic anisotropy of the as-fabricated CFO thin film to a unidirectional one upon being subjected to external electric fields, the magnetization prefers to point along [110] direction. By reversing the polarity of the applied electric field, $\mathrm{Co}^{2+}$ ion will drift back to its original position and the energetically preferred magnetization is aligned along the [ $\overline{11} 0]$ direction [Fig. 3(e)]. As such, it is confirmed that the electric field-induced migration and local redistribution of $\mathrm{Co}^{2+}$ ions account for the magnetization reversal in CFO thin films. The back-diffusion energy barrier for $\mathrm{Co}^{2}$ ${ }^{+}$cation is $\sim 0.6 \mathrm{eV}$, which is sufficient to overcome the thermal disturbance at room temperature and make the system stable. As such, the ion migration process is nonvolatile in nature, and the retention of magnetization reversal can be sustained for days. It is noteworthy that the local redistribution of $\mathrm{Co}^{2+}$ ions may also result in dielectric polarization, which may influence the magnetization reversal via a multiferroic coupling.

The modulation of magnetism with the electric fieldinduced ion migration within the magnetic structure provides the advantage of being simple and stable for large numbers of device operating cycles. The crystalline direction of the 

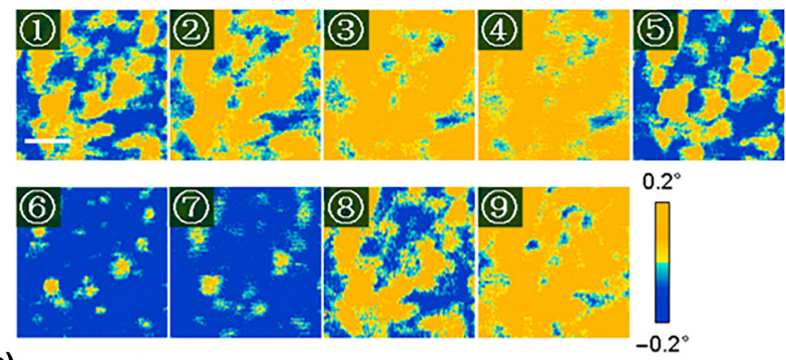

(a)

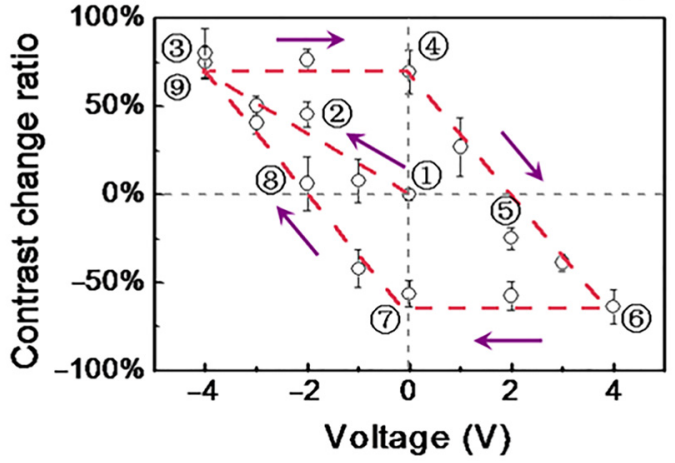

(b)

Figure 2. Reversible magnetization reversal under various electric fields. (a) MFM images of the CFO thin film at the pristine state and after being subjected to various biased voltages. Scale bar: $200 \mathrm{~nm}$. (b) Evolution of the contrast change ratio after being subjected to various biased voltages in the sequence 0 V-4 V-0 V-4 V-0 V-4 V. Adapted with permission from Ref. 23 (American Chemical Society, 2015).

ferrite film may also strongly influence the magnetization modulation as different crystal directions can have different extents of cation migration and redistribution. ${ }^{[35,36]}$ To evaluate the efficiency of ion migration and related magnetism modulation along various crystalline direction, epitaxial polycrystalline CFO sample with symmetries and orientations repeating the underneath polycrystalline SRO substrate (with a span of low to high index [hkl] crystal directions) is deposited in a grain over grain (cube-on-cube) combinatorial substrate epitaxy (CSE) fashion by PLD technique [Fig. 4(a)], ${ }^{[37]}$ which exhibits magnetization reversal behavior as shown in Figs. 4(b) and 4(c). CSE approach further confirms that although the magnetization reversal in all miscut angles from [110] direction are fully reversible, obvious decrease in the remanent magnetic contrast phase change ratio upon being subjected to either $+4 \mathrm{~V}$ or $-4 \mathrm{~V}$ stimuli is recorded as the miscut angle increases gradually [Figs. 4(d) and 4(e)]. The maximum magnetization modulation along [110] direction is consistent with the relative ease of $\mathrm{Co}^{2+}$ ion migration between the $\mathrm{B}$-site $\mathrm{Fe}^{3+}$ vacancies along the $B-B$ axis.

In order to explore the spatial uniformity of magnetization modulation via ion migration, $\mathrm{Zhu}$ et al. further correlate the observed magnetic behavior with electrical characteristics in lithium ferrite $\mathrm{LiFe}_{5} \mathrm{O}_{8}$ (LFO) thin films. LFO is also a fast ion conductor, wherein the migration of $\mathrm{Li}^{+}$ions may lead to localized resistive switching and helps understand the mechanism of magnetization modulation. In $\mathrm{LiFe}_{5} \mathrm{O}_{8}$, the $\mathrm{Li}^{+}$ ions and three-fifths of the $\mathrm{Fe}^{3+}$ ions occupy the B-site octahedral center, while the remaining $\mathrm{Fe}^{3+}$ ions occupy the center of the A site tetrahedron [Fig. 5(a)]. ${ }^{[24]} \mathrm{A}$ and $\mathrm{B}$-site $\mathrm{Fe}^{3+}$ ions are antiferromagnetically coupled. In oxygen deficient $\mathrm{LiFe}_{5} \mathrm{O}_{8-\mathrm{x}}$ samples part of the B-site $\mathrm{Fe}^{3+}$ ions are reduced to $\mathrm{Fe}^{2+}$ ions with the lower magnetic moment, and the net magnetic moment of the sample is determined by the difference of the A and B-site occupied $\mathrm{Fe}^{3+}$ ions. De-intercalation of $\mathrm{Li}^{+}$ion out of the host LFM material can result in re-oxidation of $\mathrm{Fe}^{2+}$ to $\mathrm{Fe}^{3+}$ ions, hence increasing the magnetic moment of the lithium ferrite correspondingly. The reverse process occurs during $\mathrm{Li}^{+}$ ion intercalation. Meanwhile, the $\mathrm{Li}^{+}$ion de-intercalation/intercalation process modulates the local concentration of hopping centers such as $\mathrm{Li}^{+}$vacancies in the film, which in turn can lead to resistive switching between the initial high resistance state (HRS) and low resistance state (LRS). In accordance with this hypothesis, the oxygen-deficient $\mathrm{LiFe}_{5} \mathrm{O}_{8-\mathrm{x}}$ layer sandwiched between SRO and Pt electrodes [Fig. 5(b)] exhibits three level resistive switching feature [Fig. 5(c)] and a continuous modulation of the upward-magnetized domain [Fig. 5(e)]. The dimension of the magnetic domain increases and decreases at the LRS and HRS of the resistive switching process, correspondingly, while the magnitude of the magnetization modulation is not spatially uniform, confirming that the observed magnetization reversal is ascribed to the conductive filaments growth and annihilation associated with the localized migration of $\mathrm{Li}^{+}$ion and the subsequent redox activities. Detailed analysis of the MFM image evolution also suggests that local modulation provided by $\mathrm{Li}^{+}$ion de-intercalation and intercalation lead to the local domain wall movement within a distance of $100 \mathrm{~nm}$ [Figs. 5(d) and 5(f)], which can be ascribed to the dimension increase/decrease of the domains with the magnetization direction point upwards or downwards, respectively. The overall volume change of the material and related strain effects caused by the extraction and intercalation of $\mathrm{Li}^{+}$ions from and into the LFO grains may also influence the magnetization of the sample.

The ion migration-based magnetization modulation is also reflected in the changes of the saturated magnetic moment $\left(M_{\mathrm{S}}\right)$ and coercive field $\left(H_{\mathrm{C}}\right)$ of magnetic thin film. ${ }^{[25,38-40]}$ Song et al. investigated the resistive switching and magnetic modulation in dilute magnetic semiconductors (DMS) $\mathrm{Zn}_{0.95} \mathrm{Co}_{0.05} \mathrm{O}$. It is found that the migration of oxygen ions and formation of oxygen vacancy conductive filaments yields a greater overall volume occupied by the bound magnetic polarons [Fig. 6(a)], which increases the probability of overlapping more $\mathrm{Co}^{2+}$ ions into the ferromagnetic domain and thus enhances the ferromagnetism of the DMS layer. As shown in Figs. 6(b)-6(f), accompanying the resistive switching between the HRS and LRS, both the saturated magnetization $\left(M_{\mathrm{S}}\right)$ and the coercive field $\left(H_{\mathrm{C}}\right)$ of the $\mathrm{Zn}_{0.95} \mathrm{Co}_{0.05} \mathrm{O}$ layer are greatly influenced reversibly, confirming the occurrence of ion migration induced magnetism modulation. As such, manipulating the magnetization in an electrical means without the need of 


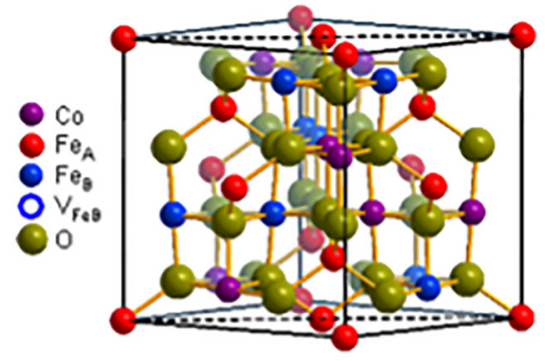

(a)

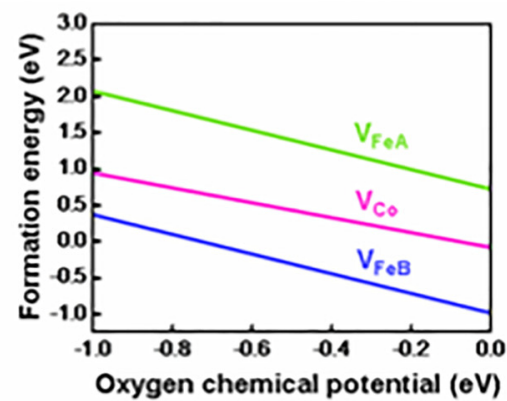

(b)

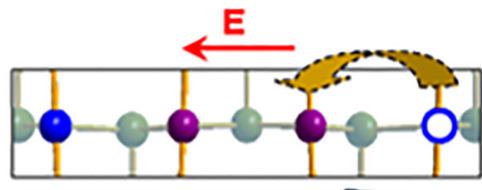

Preferred magnetization

(d)

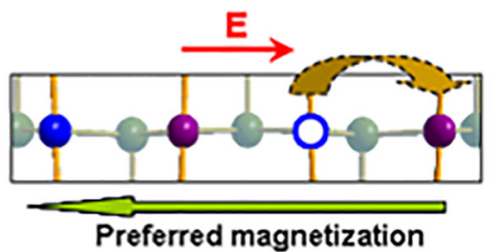

(e)

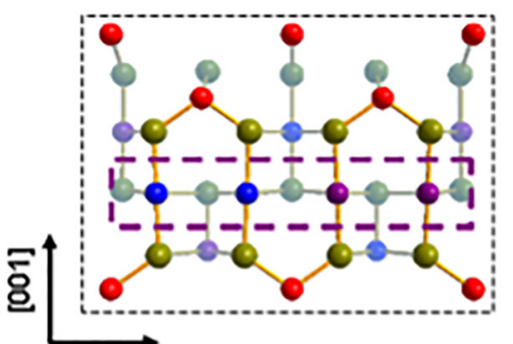

[110]

Formation energy

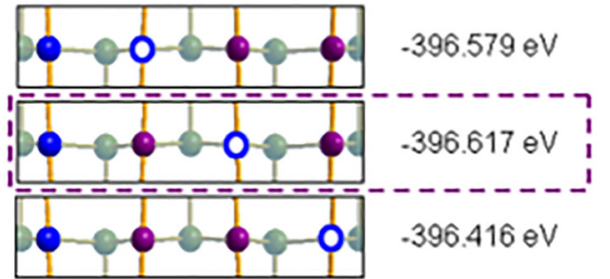

(c)

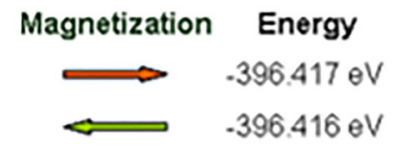

Magnetization Energy

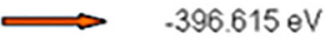

$\Longleftarrow-396.617 \mathrm{eV}$

Figure 3. Theoretical model for the electric field-induced magnetization reversal in a CFO unit cell. (a) Lattice structure of an ideal $\mathrm{CoFe} \mathrm{O}_{4}$ unit cell (left panel) and the corresponding (110) plane (right panel). The dashed box marks the distribution of the cations along the [110] direction. (b) Dependence of the formation energy of the Co vacancy, A-site Fe vacancy, and B-site Fe vacancy on the oxygen chemical potential. (c) Localized structures of the CFO unit cell with different locations of the Fe vacancy occupation. (d), (e) Localized structures and preferred magnetizations of the CFO unit cell with a Fe vacancy present along the [110] direction and with different locations of the $\mathrm{Co}$ occupation after the occurrence of electric field-induced migration of $\mathrm{Co}^{2+}$ ions. The shaded brown spheres represent oxygen atoms located outside the [110] crystal plane under evaluation. Reproduced with permission from Ref. 23. (American Chemical Society, 2015).

external magnetic field can be used to achieve a new paradigm where the interaction of spin and charge lead to faster, lowenergy, and multibit information storage applications. The utilization of ionic liquid as the gating medium further enhances the efficiency of electric field-induced modulation on complex oxide materials of $\mathrm{La}_{\mathrm{x}} \mathrm{Sr}_{1-\mathrm{x}} \mathrm{MnO}$, which shows tunable Curie temperature, enhanced saturation magnetization and obvious changes in the electrical resistance. ${ }^{[26,27,41-45]}$

\section{Reversible control of magnetism by external ion modulation}

In addition to the migration of ionic species that forms the magnetic structures, the ions from the external sources may also influence the magnetism of ferromagnetic and antiferromagnetic materials. ${ }^{[21,22,26,27]}$ For instance, due to the successful demonstration of reversible lithium ion intercalation process for thousands of charge-discharge cycles in electrochemical systems, ${ }^{[46,47]}$ Dasgupta et al. established a chemistrycontrolled yet fully reversible change in magnetic response of the spinel iron oxide $\gamma-\mathrm{Fe}_{2} \mathrm{O}_{3}$ to the $\mathrm{Li}^{+}$ion intercalation and de-intercalation processes, with $\mathrm{LiPF}_{6}$ as the external ion source in liquid state. ${ }^{[21]}$ It is shown that by carefully selecting the discharge potential, up to 1 mole $\mathrm{Li}^{+}$ion per formula unit of $\gamma-\mathrm{Fe}_{2} \mathrm{O}_{3}$ can be inserted into the iron ferrite crystal [Fig. 7(a)], which leads to large switching between distinctly different magnetic states while maintains the spinel structure intact 


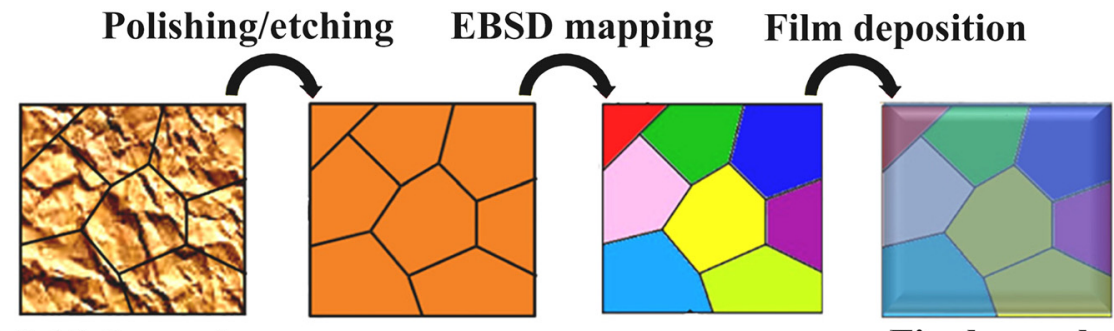

(a) Initial sample

Final sample

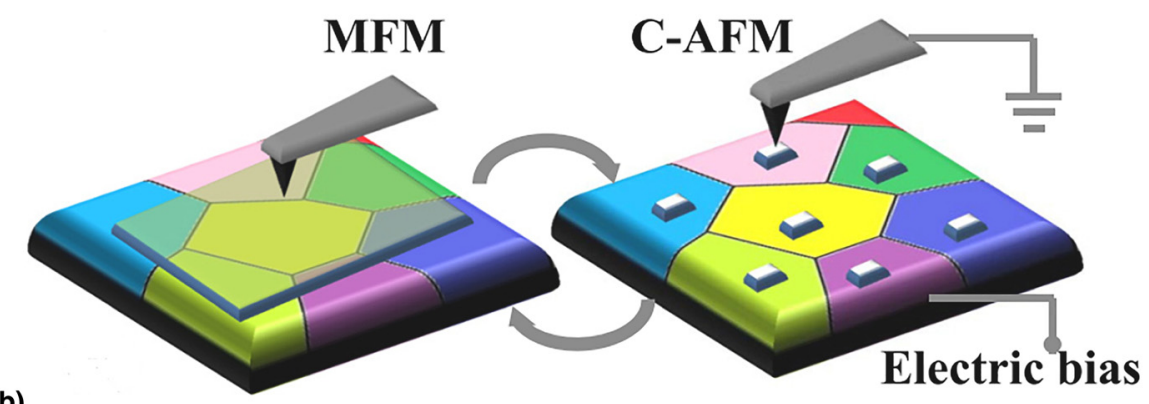

(b)
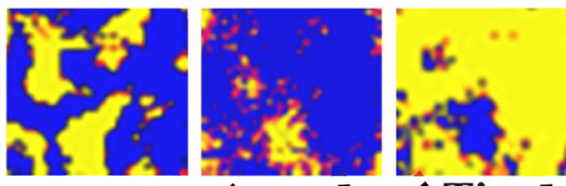

$0 \mathrm{~V}$
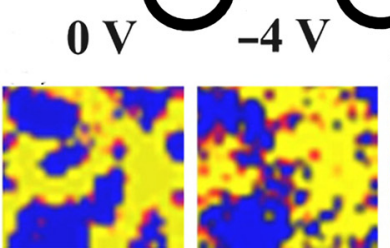

reversal

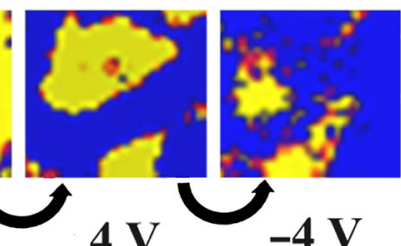

$0.2^{\circ}$

(c)
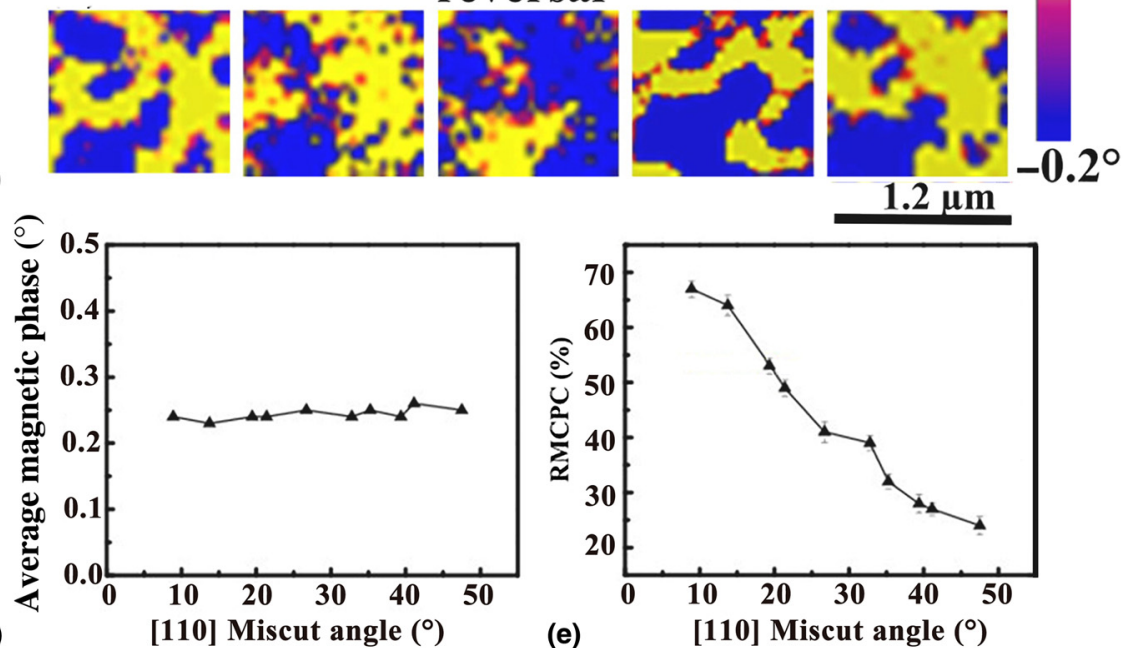

(e)

[110] Miscut angle $\left({ }^{\circ}\right)$

Figure 4. (a) Schematic of the Combinatorial Substrate Epitaxy (CSE) approach to derive the structure-property relationship at the grain scale: (1) Rough polycrystalline surface; (2) topography image of the polished surface; (3) the orientation of the substrate grains determined by Electron Backscattered Diffraction (EBSD); and (4) EBSD/Energy-dispersive x-ray spectroscopy mapping and scanning probe microscopy performed over the same region of the polycrystalline substrate after film deposition to determine the library of the structure-property relationship. (b) Illustration of MFM and C-AFM operations on the CSE samples. (c) Electric field-induced magnetization reversal on domains with the miscut angle from the [110] direction of $8.9^{\circ}$ (upper panel) and $36.6^{\circ}$ (lower panel), respectively. (d) Average magnetic phase of the domains after being subjected to $0 \mathrm{~V}-4 \mathrm{~V}-+4 \mathrm{~V}$ voltage cycles, as a function of the miscut angle from the [110] direction. (e) Average reversible magnetic contrast phase change (RMCPC) upon being subjected to either $+4 \mathrm{~V}$ or $-4 \mathrm{~V}$ stimuli, as a function of the miscut angle from the [110] direction. Reproduced with permission from Ref. 37 under the Creative Commons Attribution (CC BY) license (http://creativecommons.org/licenses/by/4.0/).

[Fig. 7(b)]. The Li intercalation-induced magnetization modulation shows two clearly discernible regimes [Fig. 7(c)]. At the initial state when the discharge potential decreases from 3.5 to $1.7 \mathrm{~V}$, the magnetization $M_{\mathrm{S}}$ rises slightly. Then a monotonic and significant decrease in $M_{\mathrm{S}}$ from 74 to 54 $\mathrm{Am}^{2} / \mathrm{kg}$ is observed. This can be ascribed to the insertion of $\mathrm{Li}^{+}$ions that reduce part of the tetrahedral $\mathrm{Fe}^{3+}$ to $\mathrm{Fe}^{2+}$ species and their subsequent rearrangement to the neighboring 
(a)
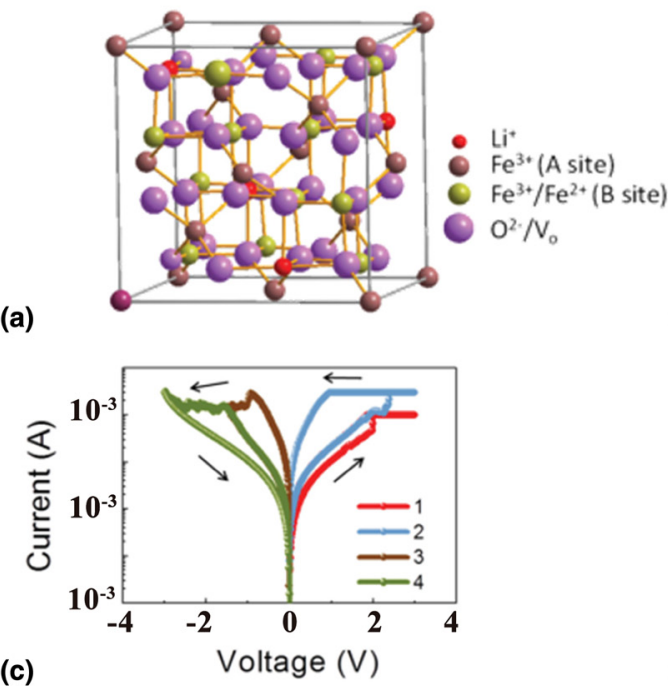

(b)
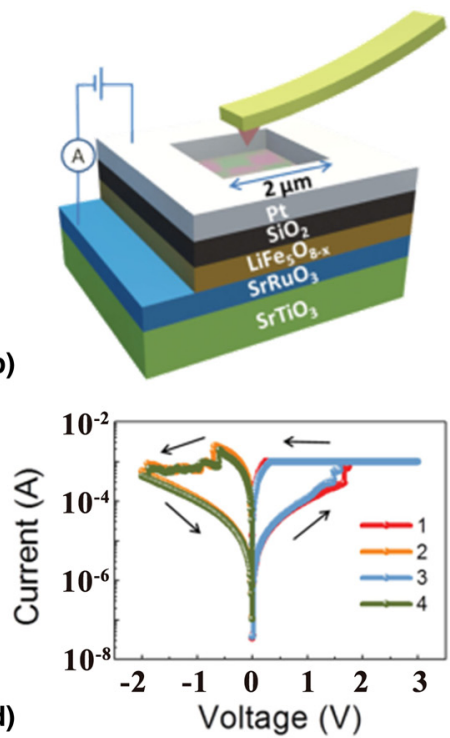

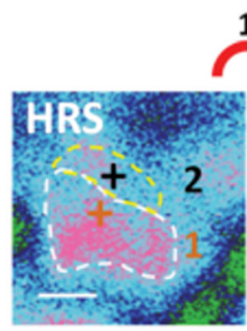

1
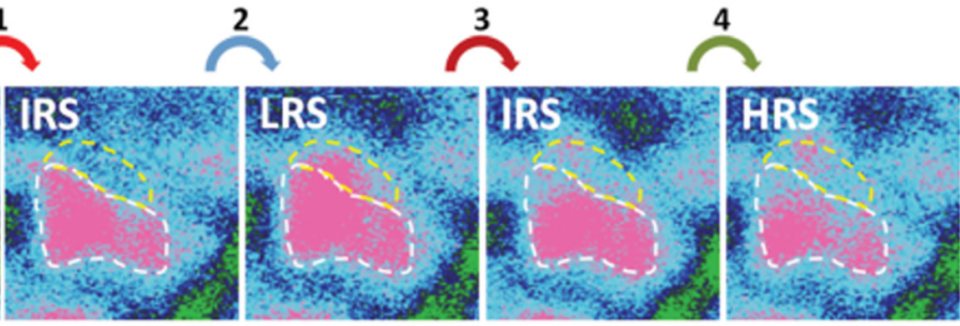

(e)
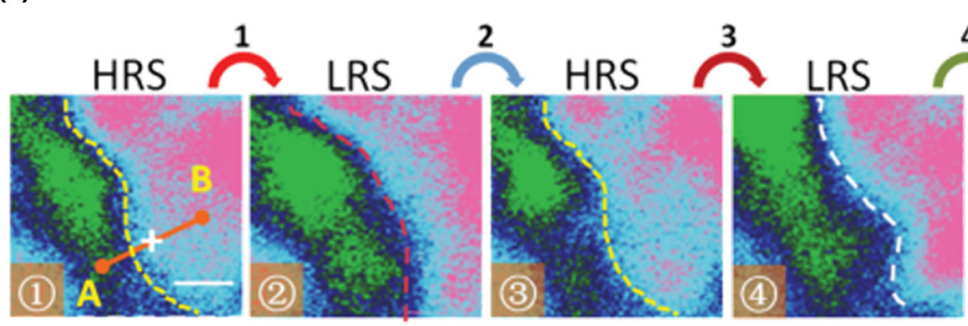

$-0.2$

0.2

(f)

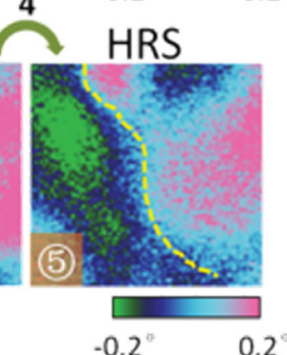

Figure 5. Reversible modulation of magnetic domain properties in Pt/LFO/SRO devices. (a) The inverse spinel-structured LiFe $\mathrm{O}_{8}$ unit cell. (b) Schematic of the measurement setup. After programming the Pt/LFO/SRO device (defined by the via structure), the MFM images were obtained without the bias voltage. (c) and (d) current-voltage characteristics of the Pt/LFO/SRO device showing consecutive and bistable resistive switching behaviors, respectively. (e) MFM images of an area with a dominant upward domain at different resistance states in (c). Scale bar: $200 \mathrm{~nm}$. (f) MFM images of an area with apparent magnetization changes, measured at different resistance states in (d). Scale bar: $200 \mathrm{~nm}$. Reproduced with permission from Ref. 24 (John Wiley and Sons, 2016).

octahedral site with more space. Net ferromagnetism increases as the B sublattice occupancy grows at the expense of A sublattice shrinkage. The change in the magnetization is fully reversible as shown in the charge-discharge cycles of Fig. 7(c). In principle, the concept of dynamic and reversible control over bulk magnetism via ion migration and electrochemical processes can be applied to any intercalation-friendly magnetic materials.

On the other hand, it is known that ferromagnetic bilayers such as $\mathrm{Co} / \mathrm{AlO}_{\mathrm{x}}$ and $\mathrm{CoFe} / \mathrm{MgO}$ exhibit strong perpendicular magnetic anisotropy (PMA) as derived from interfacial $\mathrm{Co} / \mathrm{O}$ interaction. $^{[48,49]}$ Rather than the charge accumulation and band shifting in the metal layer, ${ }^{[10,50,51]}$ modification to the oxygen stoichiometry and defect structure at the metal/metal oxide interface via electric field-driven oxygen ion migration can also tune the magnetism of the magnetic metal thin film reversibly. ${ }^{[22]}$ Bauer et al. studied the interfacial magnetism modulation of $\mathrm{Co} / \mathrm{GdO}_{\mathrm{x}}$ bilayer in a $\mathrm{Au} / \mathrm{Ta} / \mathrm{GdO} / \mathrm{Co} / \mathrm{Pt} / \mathrm{Ta}$ stacking structure [Figs. 8(a) and 8(b)] associated with the occurrence of electric field-induced migration of the oxygen ion from the metal oxide layer to the metal thin film. Scanning magneto-optical Kerr (MOKE) analysis reveals that 
(a)
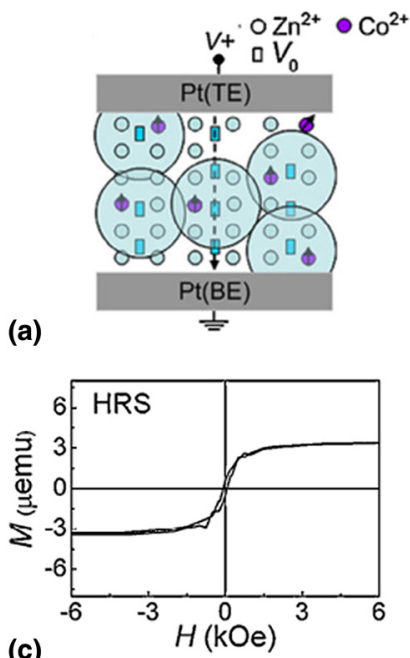

(c)
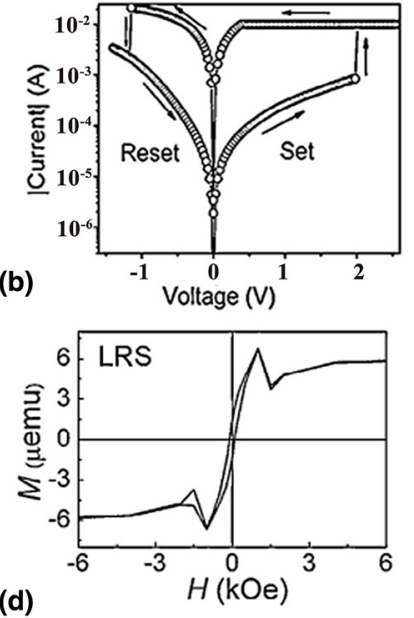

(d)
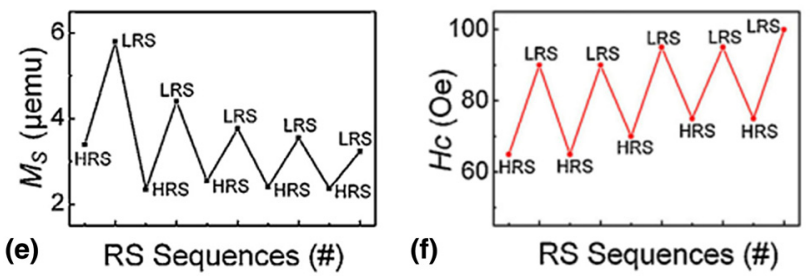

Figure 6. (a) Mechanism of the resistive switching and magnetic modulation during the set process and (b) the current-voltage curve of the Pt/ $\mathrm{Zn}_{0.95} \mathrm{CO}_{0.05} \mathrm{O} / \mathrm{Pt}$ device. The oxygen vacancy-based conductive filament is highlighted by the vertical arrow in (a). Room-temperature magnetization hysteresis loops of the $\mathrm{Pt} / \mathrm{Zn}_{0.95} \mathrm{CO}_{0.05} \mathrm{O} / \mathrm{Pt}$ device at (c) HRS and (d) LRS. The (e) saturated magnetic moment $\left(M_{\mathrm{S}}\right)$ and $(\mathrm{f})$ coercive field $\left(H_{\mathrm{c}}\right)$ are modulated reversibly during the resistive switching process. Reproduced with permission from Ref. 25 (John Wiley and Sons, 2012).

the application of $-4 \mathrm{~V}$ voltage for $240 \mathrm{~s}$ at elevated temperature of $100{ }^{\circ} \mathrm{C}$ give rise to an obvious and progressive decrease in the coercive field beneath the electrode, which can be ascribed to the over-oxidation of the Co interface and the reduction in PMA [Figs. 8(c)-8(f)]. It is noteworthy that the enhanced PMA as shown in Fig. 8(d) may also be arising from the better interaction between the $\mathrm{Co}$ and $\mathrm{Pt}$ at their interface. The PMA is completely recovered by reversing the polarity of the gating voltage to $+4 \mathrm{~V}$ at $100{ }^{\circ} \mathrm{C}$ for $270 \mathrm{~s}$ [Fig. 8(g)], allowing the voltage control of magnetism to an extent that has never before been achieved with conventional magneto-electric coupling mechanisms.

\section{Construction of magnetic conductive filaments via foreign metal ion migration and redox activities}

The key feature of nanoscale ion migration and related redox activities are the construction of discrete conductive filaments in resistive switching memory, which give rise to the bistable information storage and even in-memory computing capabilities. ${ }^{[14-16]}$ Generally, electrochemically active electrode metals (including $\mathrm{Ag}, \mathrm{Cu}, \mathrm{Co}, \mathrm{Ni}$, and, etc.) will be oxidized into cations under the application of the external electric field, and subsequently injected into the insulating layer. The cations will migrate towards the cathode through the insulating media, and get reduced to metal atoms with electrons injected from the cathode. The reduction of the cations to atoms can occur either at the insulator/cathode interface or inside the insulating later, which depends on the difference in the motilities of the cations and electrons in the insulator. The aggregation of the reduced metal atoms and continuous growth of the metal cluster will eventually form a conical shaped conductive filament that connects both the anode and cathode, leading to obvious resistance/conductance switching of the sandwich structure devices to the LRS. When the polarity of the external electric field is reversed, the conductive filament will be disrupted at its narrowest point through either Joule heating induced melting or electrochemical oxidation of the metal atoms into the cations, programming the device back to the initial HRS. Particularly, the formation and evolution of magnetic conductive filaments by the injection and migration of magnetic ions e.g., $\mathrm{Co}^{2+}$ and $\mathrm{Ni}^{2+}$ would also endow resistive switching devices more interesting magneto-ionic characteristics that supplement the modulation of magnetic properties as discussed above. For example, if the conductive filament in a ferromagnet/insulator/ferromagnet device is not connected to the cathode and the effective thickness of the residual insulating layer is thin enough for the spin-polarized tunneling of electrons across it, a magnetic tunnel junction (MJT) with a TMR phenomena would be theoretically observed. ${ }^{[52-55]}$

In 2014, Yang et al. for the first time report the construction of MTJ by the migration of $\mathrm{Co}^{2+}$ ions in $\mathrm{Co} / \mathrm{ZnO} / \mathrm{Fe}$ sandwich structures. ${ }^{[28]}$ As shown in Fig. 9(a), multi-layered films with a structure of $\mathrm{Au} / \mathrm{Co} / \mathrm{ZnO} / \mathrm{Fe} / \mathrm{Au}$ are deposited on thermally oxidized silicon substrates. The application of positively biased voltage sweep can ionize the top cobalt electrode and inject the $\mathrm{Co}^{2+}$ ions into the $\mathrm{ZnO}$ layer. After traveling a short distance within the insulating layer, the cations are reduced back to atomic forms and gradually from the metallic Co conductive filaments from the anode to the cathode [Fig. 9(b)]. By readily controlling the gap width between the magnetic conductive filament front and the bottom ferromagnetic electrodes for the spin-polarized tunneling of conduction electrons (length of the Co filament), the resistive switching device exhibits non TMR, positive TMR with the Co filament, and the Fe electrode in an anti-parallel magnetic configuration under low magnetic field, negative TMR arising from the resonant tunneling caused by impurity scattering in the $\mathrm{ZnO}$ barrier, ${ }^{[56]}$ and non TMR phenomena in its HRS, intermediate resistance states, and LRS, respectively [Figs. 9(c)-9(f)]. In addition to the magnetization modulation with the native ions presenting in the ferrite thin films, the construction of magnetic tunneling junction and related TMR phenomena through ion migration in resistive switching cells with ferromagnetic electrodes may provide a more versatile platform for building novel sensors and information storage devices. Nevertheless, it is noteworthy that oxidization of the magnetic metal (such as $\mathrm{Co}$ or $\mathrm{Fe}$ ) electrodes 

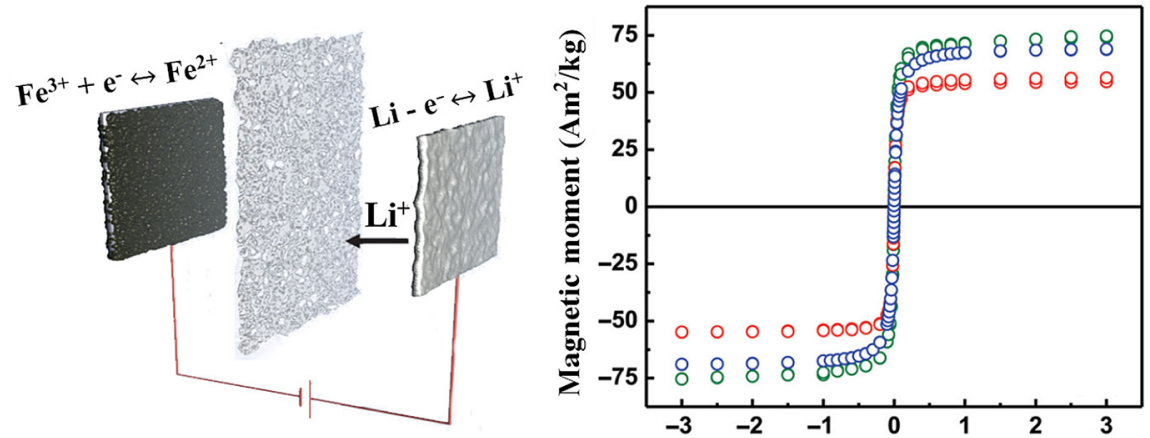

(a)

(b)

Magnetic field (T)

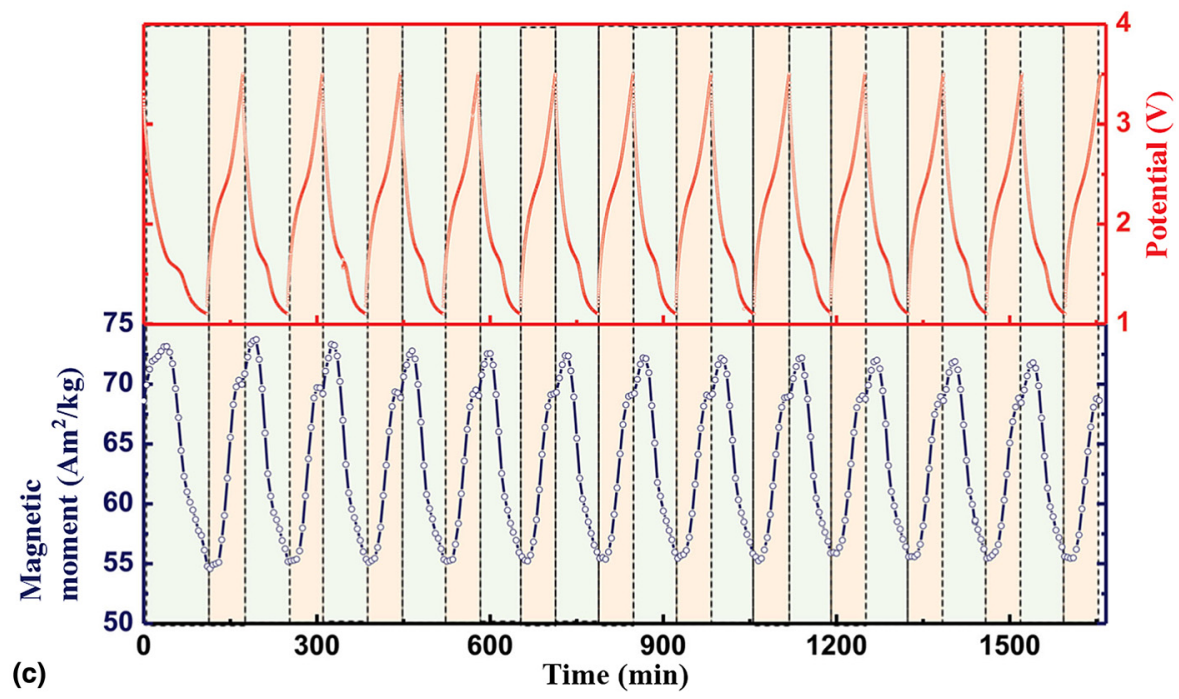

Figure 7. (a) Schematic of the typical electrochemical cell showing reversible $\mathrm{Li}^{+}$ion intercalation and de-intercalation with spinel iron ferrite $\gamma-\mathrm{Fe}_{2} \mathrm{O}_{3}$. (b) Magnetic hysteresis measured at different stages of lithiation: blue, green, and red symbols stand for the as-prepared $\gamma-\mathrm{Fe}_{2} \mathrm{O}_{3}$ sample, and the samples that are discharged down to 1.7 and $1.1 \mathrm{~V}$, respectively. after reaching the desired discharge potential, the electrochemical cell has been electrically disconnected prior to the magnetic measurements. (c) Fully-reversible variation in magnetic response (measured at the magnetic field of $1 \mathrm{~T}$ ) of the sample. The discharge and charge cycles are carried out with a potentiometric constant current pf $115 \mathrm{~mA} / \mathrm{g}$ and cut-off potentials of 1.1 and $3.5 \mathrm{~V}$ versus $\mathrm{Li}^{+} / \mathrm{Li}$ during lithiation and delithiation steps, respectively. Reproduced with permission from Ref. 21 (John Wiley and Sons, 2014).

may also lead to the modification to the oxygen stoichiometry and defect structure at the metal/metal oxide interface, which tunes the magnetism of the magnetic metal thin film reversibly.

Inspired by the above work, Otsuka and Long et al. constructed ferromagnetic conductive filaments in resistive switching devices with $\mathrm{Co}^{2+}$ or $\mathrm{Ni}^{2+}$ ions, and explored the AMR behavior of the $\mathrm{Ni} / \mathrm{TiO}_{2} / \mathrm{Pt}$ or $\mathrm{Co} / \mathrm{HfO}_{2} / \mathrm{Pt}$ structure in their LRS [Figs. 10(a) and 10(b)]. ${ }^{[29-31]}$ It is found that the $\mathrm{Ni} /$ $\mathrm{TiO}_{2} / \mathrm{Pt}$ device shows convexity in its AMR plot when the direction of the magnetic field is perpendicular to the direction electric current flowing across the device [Figs. 10(c) and $10(d)]$, whereas a concavity is observed when the magnetic field is aligned in the direction parallel to that of the device currents [Fig. 10(e)]. ${ }^{[29,30]}$ This is consistent with the fact that AMR occurred in ferromagnetic transition metal depends strongly on the direction of the magnetization with respect to the electric current direction. ${ }^{[57,58]}$ By rotating the direction of the out-of-plane magnetic field with respect to the substrate surface with a fixed magnitude of $2 \mathrm{~T}$ in the LRS of the $\mathrm{Co} /$ $\mathrm{HfO}_{2} / \mathrm{Pt}$ device [Fig. 10(f)], it is demonstrated that the spontaneous magnetization of the electroformed Co conductive filament, showing maximum magnetoresistance in the resistance-rotating angle curve [Fig. $10(\mathrm{~g})$ ], is tilted by $30^{\circ}$ from the device current direction. As such, the construction of ferromagnetic conductive filaments with clear magnetic structures is not only beneficial for making a multilevel memory with both resistive switching and spin dependences but also useful to reveal their detailed magnetic structure information.

\section{Conclusion and future outlook}

The electric field-induced ion migration in resistive switching devices provides a simple yet versatile approach for modulating the magnetism of the functional materials. Migration of ionic specie that either constitutes the magnetic structure or comes from the external sources can effectively lead to the 


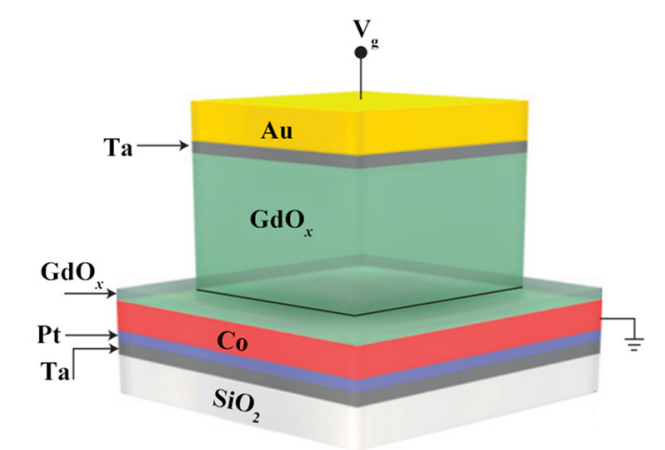

(a)

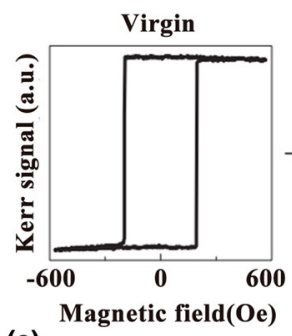

(c)

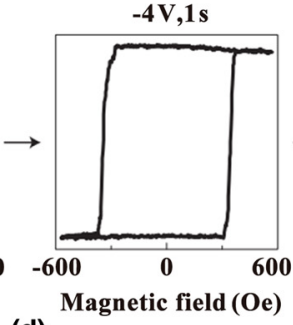

(d)

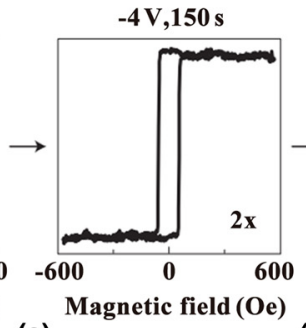

(e)

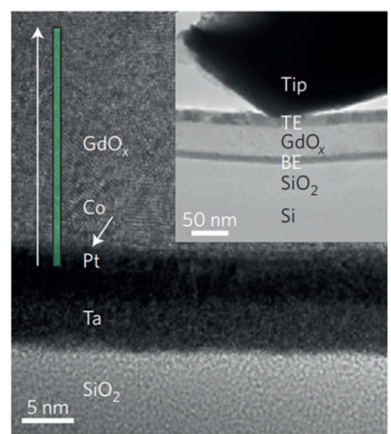

(b)

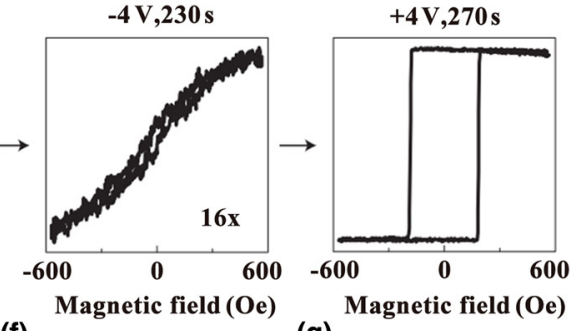

(g)

Figure 8. (a) Device schematic and (b) high-resolution cross-sectional transmission electron microscopic image of the Au/Ta/GdO $/ \mathrm{Co} / \mathrm{Pt} / \mathrm{Ta}$ multilayer structure for the electric field-controlled magnetic anisotropy. Polar magneto-optical Kerr (MOKE) hysteresis loops of the sample measured at room temperature at the centre of the gate electrode showing the device in its virgin state (c), after applying $V_{\mathrm{g}}=-4 \mathrm{~V}$ at $100{ }^{\circ} \mathrm{C}$ for (d) $1 \mathrm{~s}$, (e) $150 \mathrm{~s}$ and (f) $230 \mathrm{~s}$, and (g) after applying $V_{\mathrm{g}}=4 \mathrm{~V}$ at $100^{\circ} \mathrm{C}$ for $270 \mathrm{~s}$. Note that the Kerr signal intensity in (e) is reduced by a factor of 2 and in (f) by a factor of 16 , as indicated by inset number. Reproduced with permission from Ref. 22 (Springer Nature, 2014).

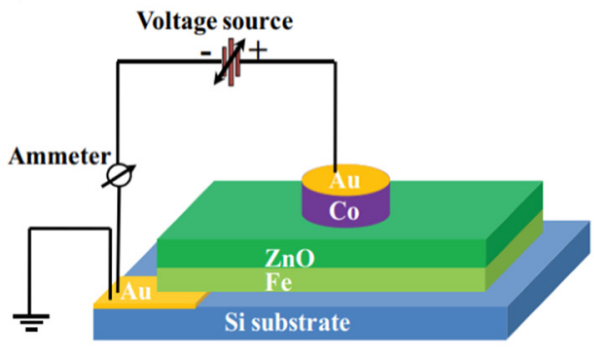

(a)

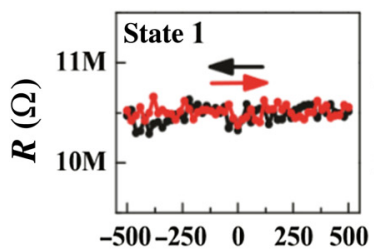

(c)

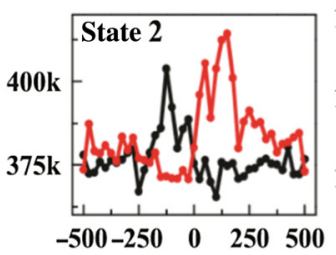

(d)

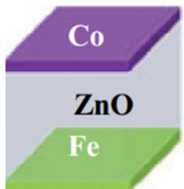

State 1

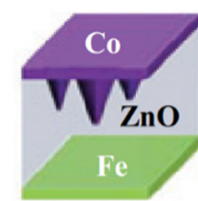

State 3

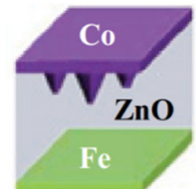

State 2

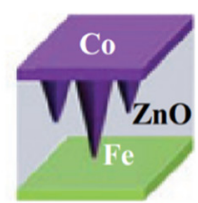

State 4

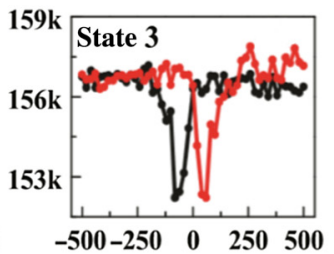

(e)

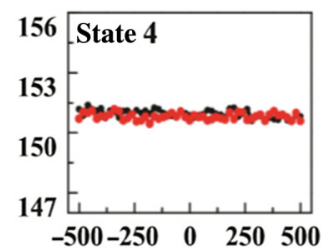

(f)

$H$ (Oe)

Figure 9. (a) Schematic of the multi-layered resistive switching $\mathrm{Co} / \mathrm{ZnO} / \mathrm{Fe}$ structures. (b) Evolutional formation of the $\mathrm{Co}$ conductive filament in the $\mathrm{Co} / \mathrm{ZnO} / \mathrm{Fe}$ structure under the positively biased voltage applied to the top cobalt electrode. The tunneling magnetoresistance (TMR) of the CoZnO/Fe structures measured in (c) State 1, (d) State 2, (e) State 3 and (f) State 4. Reproduced with permission from Ref. 28 (Institute of Physics (IOP), 2014). 


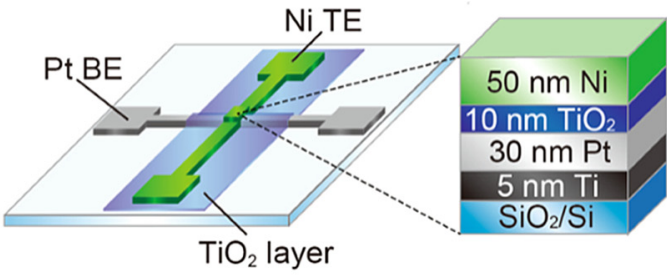

(a)

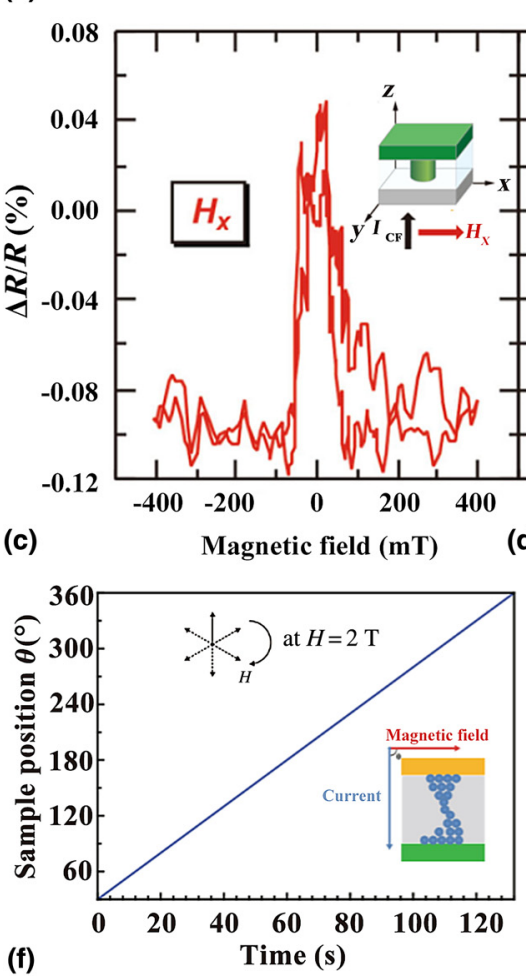

(b)

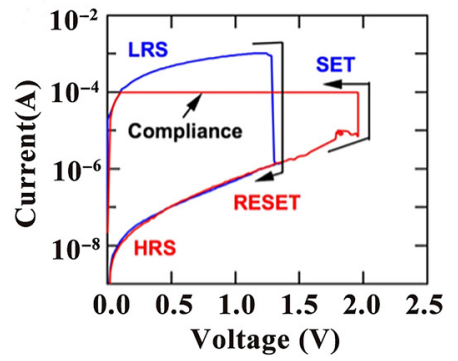

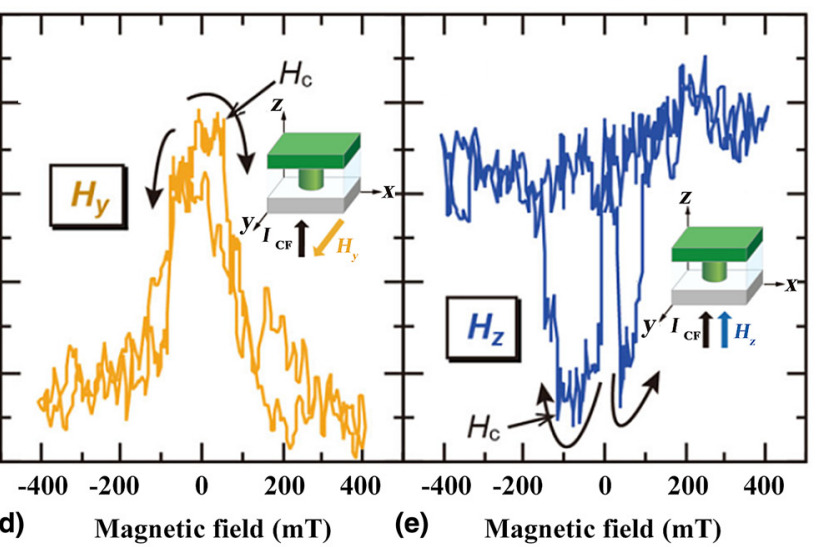

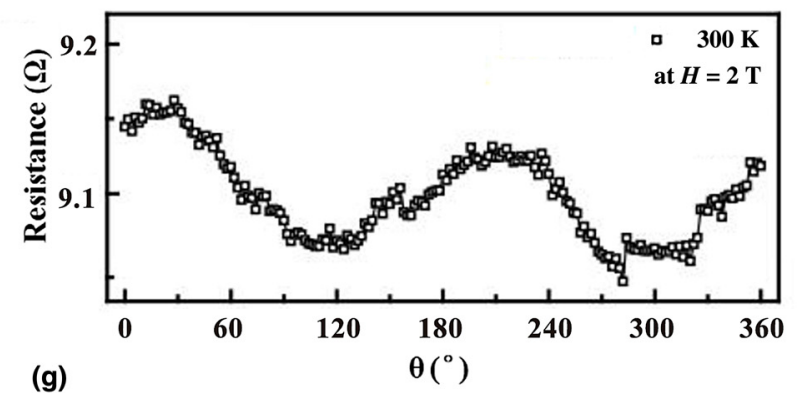

Figure 10. (a) Schematics and cross-section of the crossbar $\mathrm{Ni}_{\mathrm{TiO}} / \mathrm{Pt}$ device structure. (b) The typical current-voltage curve of the $\mathrm{Ni} / \mathrm{TiO} \mathrm{O}_{2} / \mathrm{Pt}$ device showing

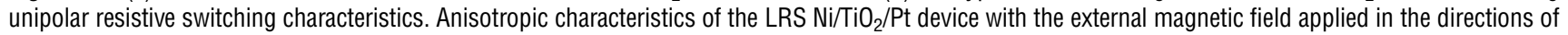
(c) $H_{x}$, (d) $H_{y}$ and (e) $H_{z}$, respectively. (f) Scanning of the out-of-plane magnetic field direction with respect to the substrate surface and (g) the related room-temperature magnetoresistance of the $\mathrm{Co} / \mathrm{HfO}_{2} / \mathrm{Pt}$ device as a function of the rotating angle. (a)-(e) are reproduced with permission from Ref. 29 (Springer Nature, 2014). (f) and (g) are reproduced with permission from Ref. 31 under the Creative Commons Attribution (CC BY) license (http:// creativecommons.org/licenses/by/4.0/).

magnetization modulation in ferromagnetic thin films, which is useful for realizing low power spintronic devices. ${ }^{[69]}$ The construction of foreign magnetic conductive filaments in the insulating matrix also gives rise to TMR and AMR characteristics. Recently it is demonstrated that by accurately controlling the evolution dynamics and thus the dimension of the conductive filaments, NPC with quantized conductance characteristics can be obtained in resistive switching devices. The stepwise development of the device conductance in the unit of $G_{0}=$ $77.5 \mathrm{uS}$ greatly decorates the bistable switching features of memory devices, and allows for the bottom-up design of quantum information processing and neuromorphic computing architectures through the higher-logic algorithm. ${ }^{[59-65]}$ On the other hand, the ballistic magnetoresistance effect discovered in 1999 significantly stimulates the upsurge of research on the submicron ferromagnetic nanostructure. ${ }^{[66-68]}$ In case that this anomalous giant magnetoresistance effect can be reproduced precisely in the quantized conductance states of the magnetic NPC formed by $\mathrm{Co}^{2+}$ or $\mathrm{Ni}^{2+}$ migration in resistive switching memories, integration of multiple functions such as sensing, data processing, and storage into a single cell may be realized for the settlement of the von Neumann bottleneck issue encountered by the modern computer systems.

\section{Acknowledgments}

The authors acknowledge the financial support from the National Key R\&D Program of China (2017YFB0405604 and 2016YFA0201102), the National Natural Science 
Foundation of China (61722407, 61674153, 51525103, 61704178, 11474295, and 51472210), China Postdoctoral Science Foundation (2016LH0050 and 2017M610379), K. C. Wong Education Foundation (rczx0800), the Natural Science Foundation of Zhejiang Province (LR17E020001), the Provincial Natural Science Foundation of Hunan (2018JJ4037), Ningbo Science and Technology Innovation Team (2015B11001), and Key Laboratory of Advanced Materials of Ministry of Education (2017AML04).

\section{References}

1. C. Chappert, A. Fert, and F.N. Van Dau: The emergence of spin electronics in data storage. Nat. Mater. 6, 813 (2007).

2. S. Mangin, D. Ravelosona, J.A. Katine, M.J. Carey, B.D. Terris, and E. E. Fullerton: Current-induced magnetization reversal in nanopillars with perpendicular anisotropy. Nat. Mater. 5, 210 (2006).

3. R.O. Cherifi, V. Ivanovskaya, L.C. Phillips, A. Zobelli, I.C. Infante, E. Jacquet, V. Garcia, S. Fusil, P.R. Briddon, N. Guiblin, A.A. Ünal, F. Kronast, S. Valencia, B. Dkhil, and A. Barthélémy: Electric-field control of magnetic order above room temperature. Nat. Mater. 13, 345 (2014).

4. T.A. Ostler, J. Barker, R.F.L. Evans, R.W. Chantrell, U. Atxitia, 0. Chubykalo-Fesenko, S. El Moussaoui, L. Le Guyader, E. Mengotti, L. J. Heyderman, F. Nolting, A. Tsukamoto, A. Itoh, D. Afanasiev, B. A. Ivanov, A.M. Kalashnikova, K. Vahaplar, J. Mentink, A. Kirilyuk, T. Rasing, and A.V. Kimel: Ultrafast heating as a sufficient stimulus for magnetization reversal in a ferrimagnet. Nat. Commun. 3, 666 (2012).

5. R. Ramesh and N.A. Spaldin. Multiferroics: progress and prospects in thin films. Nat. Mater. 6, 21 (2007).

6. H.N. Hur, S. Park, P.A. Sharma, J.S. Ahn, S. Guha, and S.-W. Cheong: Electric polarization reversal and memory in a multiferroic material induced by magnetic fields. Nature 429, 392 (2004).

7. G. Radaelli, D. Petti, E. Plekhanov, I. Fina, P. Torelli, B.R. Salles, M. Cantoni, C. Rinaldi, D. Gutiérrez, G. Panaccione, M. Varela, S. Picozzi, J. Fontcuberta, and R. Bertacco: Electric control of magnetism at the $\mathrm{Fe} / \mathrm{BaTiO}_{3}$ interface. Nat. Commun. 5, 3404 (2014).

8. D. Chiba, M. Yamanouchi, F. Matsukura, and H. Ohno: Electrical manipulation of magnetization reversal in a ferromagnetic semiconductor. Science 301, 943 (2003).

9. Y. Yamada, K. Ueno, T. Fukumura, H.T. Yuan, H. Shimotani, Y. Iwasa, L. Gu, S. Tsukimoto, Y. Ikuhara, and M. Kawasaki: Electrically induced ferromagnetism at room temperature in cobalt-doped titanium dioxide. Science 332, 1065 (2011).

10. T. Maruyama, Y. Shiota, T. Nozaki, K. Ohta, N. Toda, M. Mizuguchi, A. A. Tulapurkar, T. Shinjo, M. Shiraishi, S. Mizukami, Y. Ando, and Y. Suzuki: Large voltage-induced magnetic anisotropy change in a few atomic layers of iron. Nat. Nano. 4, 158 (2009).

11. M. Ghidini, R. Pellicelli, J.L. Prieto, X. Moya, J. Soussi, J. Briscoe, S. Dunn, and N.D. Mathur: Non-volatile electrically-driven repeatable magnetization reversal with no applied magnetic field. Nat. Commun. 4, 1453 (2013).

12. Y.S. Chai, S. Kwon, S.H. Chun, I. Kim, B.-G. Jeon, K.H. Kim, and S. Lee: Electrical control of large magnetization reversal in a helimagnet. Nat. Commun. 5, 4208 (2014).

13. F.A. Cuellar, Y.H. Liu, J. Salafranca, N. Nemes, E. Iborra, G. Sanchez-Santolino, M. Varela, M. Garcia Hernandez, S. Okamoto, S. J. Pennycook, M. Bibes, A. Barthélémy, S.G.E. te Velthuis, Z. Sefrioui, C. Leon, and J. Santamaria: Reversible electric-field control of magnetization at oxide interfaces. Nat. Commun. 5, 4215 (2014).

14. J. Maier. Nanoionics: ion transport and electrochemical storage in confined systems. Nat. Mater. 4, 805 (2005).

15. R. Waser and M. Aono: Nanoionics-based resistive switching memories. Nat. Mater. 6, 833 (2007).

16. J.J. Yang, D.B. Strukov, and D.R. Stewart: Memristive devices for computing. Nat. Nano. 8, 13 (2013).
17. Y. Yang, P. Gao, S. Gaba, T. Chang, X. Pan, and W. Lu: Observation of conducting filament growth in nanoscale resistive memories. Nat. Commun. 3, 732 (2012)

18. D.-H. Kwon, K.M. Kim, J.H. Jang, J.M. Jeon, M.H. Lee, G.H. Kim, X.-S. Li, G.-S. Park, B. Lee, S. Han, M. Kim, and C.S. Hwang: Atomic structure of conducting nanofilaments in $\mathrm{TiO}_{2}$ resistive switching memory. Nat. Nano. 5, 148 (2010).

19. H. Tan, G. Liu, H.L. Yang, X.H. Yi, L. Pan, J. Shang, S.B. Long, M. Liu, Y. H. Wu, and R.-W. Li: Light-gated memristor with integrated logic and memory functions. ACS. Nano. 11, 11298 (2017).

20. W.H. Xue, G. Liu, Z.C. Zhong, Y.H. Dai, J. Shang, Y.W. Liu, H.L. Yang, X H. Yi, H.W. Tan, L. Pan, S. Gao, J. Ding, X.-H. Xu, and R.-W. Li: A 1D vanadium dioxide nanochannel constructed via electric-field-induced ion transport and its superior metal-insulator transition. Adv. Mater. 29, 39 (2017).

21. S. Dasgupta, B. Das, M. Knapp, R.A. Brand, H. Ehrenberg, R. Kruk, and $H$. Hahn: Intercalation-driven reversible control of magnetism in bulk ferromagnets. Adv. Mater. 26, 4639 (2014).

22. U. Bauer, L. Yao, A.J. Tan, P. Agrawal, S. Emori, H.L. Tuller, S. Dijken, and G.S.D. Beach: Magneto-ionic control of interfacial magnetism. Nat. Mater. 14, 174 (2015)

23. X. Chen, X. Zhu, W. Xiao, G. Liu, Y.P. Feng, J. Ding, and R.-W. Li: Nanoscale magnetization reversal caused by electric field-induced ion migration and redistribution in cobalt ferrite thin films. ACS. Nano. 9, 4210 (2015)

24. X. Zhu, J. Zhou, L. Chen, S. Guo, G. Liu, R.-W. Li, and W.D. Lu: In situ nanoscale electric field control of magnetism by nanoionics. Adv. Mater. 28, 7658 (2016).

25. G. Chen, C. Song, C. Chen, S. Gao, F. Zeng, and F. Pan: Resistive switching and magnetic modulation in cobalt-doped ZnO. Adv. Mater. 24, 3515 (2012).

26. B. Cui, C. Song, G. Wang, Y. Yan, J. Peng, J. Miao, H. Mao, F. Li, C. Chen, F. Feng, and F. Pan: Reversible ferromagnetic phase transition in electrode-gated manganites. Adv. Funct. Mater. 24, 7233 (2014).

27. B. Cui, C. Song, G A. Gehring, F. Li, G. Wang, C. Chen, J. Peng, H. Mao, F. Zeng, and F. Pan: Electrical manipulation of orbital occupancy and magnetic anisotropy in manganites. Adv. Funct. Mater. 25, 864 (2015).

28.Z. Yang, Q. Zhan, X. Zhu, Y. Liu, H. Yang, B. Hu, J. Shang, L. Pan, B. Chen, and R.-W. Li: Tunneling magnetoresistance induced by controllable formation of $\mathrm{Co}$ filaments in resistive switching $\mathrm{Co} / \mathrm{ZnO} / \mathrm{Fe}$ structures. EPL. 108, 58004 (2014).

29. S. Otsuka, Y. Hamada, T. Shimizu, and S. Shingubara: Ferromagnetic nano-conductive filament formed in $\mathrm{Ni} / \mathrm{TiO}_{2} / \mathrm{Pt}$ resistive-switching memory. Appl. Phys. A. 118, 613 (2015).

30.S. Otsuka, Y. Hamada, D. Ito, T. Shimizu, and S. Shingubara: Magnetoresistance of conductive filament in $\mathrm{Ni} / \mathrm{HfO}_{2} / \mathrm{Pt}$ resistive switching memory. Jpn. J. Appl. Phys. 54, 05ED02 (2015).

31. L. Li, Y. Liu, J. Teng, S. Long, Q. Guo, M. Zhang, Y. Wu, G. Yu, Q. Liu, H. Lv, and M. Liu: Anisotropic magnetoresistance of nano-conductive filament in $\mathrm{Co} / \mathrm{HfO}_{2} / \mathrm{Pt}$ resistive switching memory. Nanoscale. Res. Lett. 12, 210 (2017).

32.X.J. Zhu, C.S. Ong, X. Xu, B. Hu, J. Shangm, H. Yang, S. Katlakunta, Y. Liu, X. Chen, L. Pan, J. Ding, and R.-W. Li: Direct observation of lithium-ion transport under an electrical field in $\mathrm{Li}_{x} \mathrm{CoO}_{2}$ nanograins. Sci. Rep. 3, 1084 (2012).

33. H.-S.P. Wong, H.-Y. Lee, S. Yu, Y-S. Chen, Y. Wu, P-S. Chen, B. Lee, F. T. Chen, and M.-J. Tsai: Metal-oxide RRAM. Proc. IEEE 100, 1951 (2012).

34. I. Valov: Redox-based resistive switching memories (ReRAMs): electrochemical systems at the atomic scale. ChemElectroChem 1, 26 (2014).

35. R. Waser, R. Dittmann, G. Staikov, and K. Szot: Redox-based resistive switching memories-nanoionic mechanisms, prospects, and challenges. Adv. Mater. 21, 2632 (2009).

36. Y. Zhang, A.M. Schultz, L. Li, H. Chien, P.A. Salvador, and G.S. Rohrer: Combinatorial substrate epitaxy: a high-throughput method for determining phase and orientation relationships and its application to $\mathrm{BiFeO}_{3} / \mathrm{TiO}_{2}$ heterostructures. Acta. Mater. 60, 6486 (2012).

37. P. Dhanapal, S. Guo, B. Wang, and R.-W. Li: High-throughput investigation of orientations effect on nanoscale magnetization reversal in cobalt ferrite thin films induced by electric field. Appl. Phys. Lett. 111, 162401 (2017). 
38. H. Ohno, D. Chiba, F. Matsukura, T. Omiya, E. Abe, T. Dietl, Y. Ohno, and K. Ohtani: Electric-field control of ferromagnetism. Nature 408, 944 (2000).

39.D. Chiba, M. Sawicki, Y. Nishitani, F. Matsukura, and H. Ohno: Magnetization vector manipulation by electric fields. Nature 455, 515 (2008).

40.I. Stolichnov, S.W.E. Riester, H.J. Trodahl, N. Setter, A.W. Rushforth, K. W. Edmonds, R.P. Campion, C.T. Foxon, B.L. Gallagher, and T. Jungwirth: Non-volatile ferroelectric control of ferromagnetism in (Ga, Mn)As. Nat. Mater. 7, 464 (2008).

41.D. Chiba: Ono T. Control of magnetism in Co by an electric field. J. App.I Phys. 46, 213001 (2013).

42. L. Herrera Diez, A. Bernand-Mantel, L. Vila, P. Warin, A. Marty, S. Ono, D. Givord, and L. Ranno: Electric-field assisted depinning and nucleation of magnetic domain walls in $\mathrm{FePt} / \mathrm{Al}_{2} \mathrm{O}_{3}$ /liquid gate structures. Appl. Phys. Lett. 104, 082413 (2014).

43. B. Cui, C. Song, G.-Y. Wang, Y.-N. Yan, J.-J. Peng, J.-H. Miao, H.-J. Mao, F. Li, C. Chen, F. Zeng, and F. Pan: Reversible ferromagnetic phase transition in electrode-gated manganites. Adv. Func. Mater. 24, 7233 (2014).

44. Y.-Y. Wang, C. Song, B. Cui, G.Y. Wang, F. Zeng, and F. Pan: Room-temperature perpendicular exchange coupling and tunneling anisotropic magnetoresistance in an antiferromagnet-based tunnel junction. Phys. Rev. Lett. 109, 137201 (2012).

45. P.-X. Zhang, G.-F. Yin, Y.-Y. Wang, C. Bin, P. Feng, and S. Cheng: Electrical control of antiferromagnetic metal up to $15 \mathrm{~nm}$. Science China Physics. 59, 687511 (2016)

46. G.-N. Zhu, H.-J. Liu, J.-H. Zhuang, C.-X. Wang, Y.-G. Wang, and Y.-Y. Xia: Carbon-coated nano-sized $\mathrm{Li}_{4} \mathrm{Ti}_{5} \mathrm{O}_{12}$ nanoporous micro-sphere as anode material for high-rate lithium-ion batteries. Ener. Env. Sci. 4, 4016 (2011).

47.C. Sun, S. Rajasekhara, J.B. Goodenough, and F. Zhou: Monodisperse porous LiFePO4 microspheres for a high power Li-ion battery cathode. J. Am. Chem. Soc. 133, 2132 (2011).

48. A. Manchon, S. Pizzini, J. Vogel, V. Uhlîr, L. Lombard, C. Ducruet, S. Auffret, B. Rodmacq, B. Dieny, M. Hochstrasser, and G. Panaccione: $\mathrm{X}$-ray analysis of the magnetic influence of oxygen in $\mathrm{Pt} / \mathrm{Co} / \mathrm{AlO} \mathrm{O}_{\mathrm{x}}$ trilayers. J. Appl. Phys. 103, 07A912 (2008).

49. B. Rodmacq, A. Manchon, C. Ducruet, S. Auffret, and B. Dieny: Influence of thermal annealing on the perpendicular magnetic anisotropy of $\mathrm{Pt} / \mathrm{Co}$ / AlOx trilayers. Phys. Rev. B. 79, 024423 (2009).

50. Y. Shiota, T. Nozaki, F. Bonell, S. Murakami, T. Shinjo, and Y. Suzuki: Induction of coherent magnetization switching in a few atomic layers of FeCo using voltage pulses. Nat. Mater. 11, 39 (2012).

51.W.G. Wang, M. Li, S. Hageman, and C.L. Chien: Electric-field-assisted switching in magnetic tunnel junctions. Nat. Mater. 11, 64 (2012).

52. T. Miyazaki, and N. Tezuka: Giant magnetic tunneling effect in $\mathrm{Fe} / \mathrm{Al}_{2} \mathrm{O}_{3} / \mathrm{Fe}$ junction. J. Magn. Magn. Mater. 139, L231 (1995).

53. J.S. Moodera, L.R. Kinder, T.M. Wong, and R. Meservey: Large magnetoresistance at room temperature in ferromagnetic thin film tunnel junctions. Phys. Rev. Lett. 74, 3273 (1995).

54. S.S.P. Parkin, C. Kaiser, A. Panchula, P.M. Rice, B. Hughes, M. Samant, and S.-H. Yang: Giant tunnelling magnetoresistance at room temperature with MgO (100) tunnel barriers. Nat. Mater. 3, 862 (2004).

55. S. Yuasa, T. Nagahama, A. Fukushima, Y. Suzuki, and K. Ando: Giant room-temperature magnetoresistance in single-crystal Fe/MgO/Fe magnetic tunnel junctions. Nat. Mater. 3, 868 (2004).

56. D.S. Jeong, R. Thomas, R.S. Katiyar, J.F. Scott, H. Kohlstedt, A. Petraru, and C.S. Hwang: Emerging memories: resistive switching mechanism and current status. Rep. Prog. Phys. 75, 076502 (2012).

57.J. Smit: Magnetoresistance of ferromagnetic metals and alloys at low temperatures. Phys. 17, 612 (1951).

58. T. McGuire and R.L. Potter: Anisotropic magnetoresistance in ferromagnetic 3d alloys. IEEE. Trans. Magn. 11, 1018 (1975).

59. T. Hasegawa, K. Terabe, T. Tsuruoka, and M. Aono: Atomic switch: atom/ ion movement controlled devices for beyond Von-Neumann computers. Adv. Mater. 24, 252 (2012).

60. X. Zhu, W. Su, Y. Liu, B. Hu, L. Pan, W. Lu, J. Zhang, and R.-W. Li: Observation of conductance quantization in oxide-based resistive switching memory. Adv. Mater. 24, 3941 (2012).
61. A. Mehonic, A. Vrajitoarea, and S. Cueff: Quantum conductance in silicon oxide resistive memory devices. Sci. Rep. 3, 2708 (2013).

62. S. Long, L. Perniola, C. Cagli, J. Buckley, X. Lian, E. Miranda, F. Pan, M. Liu, and J. Suñé: Voltage and power-controlled regimes in the progressive unipolar RESET transition of $\mathrm{HfO}_{2}$-based RRAM. Sci. Rep. $\mathbf{3}$, 2929 (2013).

63. S.R. Nandakumar, M. Minvielle, S. Nagar, C. Dubourdieu, and B. Rajendran: A $250 \mathrm{mv} \mathrm{Cu} / \mathrm{SiO}_{2} / \mathrm{W}$ memristor with half-integer quantum conductance states. Nano. Lett. 16, 1602 (2016).

64. A. Wedig, M. Luebben, and D.Y. Cho: Nanoscale cation motion in $\mathrm{TaO}_{\mathrm{x}}$ $\mathrm{HfO}_{x}$ and $\mathrm{TiO}_{x}$ memristive systems. Nat. Nano. 11, 67 (2016).

65. K. Krishnan, M. Muruganathan, and T. Tsuruoka: Highly reproducible and regulated conductance quantization in a polymer-based atomic switch. Adv. Funct. Mater. 27, 10 (2017).

66. N. Garcia, M. Munoz, and Y.W. Zhao: Magnetoresistance in excess of $200 \%$ in ballistic Ni nanocontacts at room temperature and 1000 e. Phys. Rev. Lett. 82, 2923 (1999).

67.S.H. Chung, M. Munoz, N. García, W.F. Egelhoff, and R.D. Gomez: Universal scaling of ballistic magnetoresistance in magnetic nanocontacts. Phys. Rev. Lett. 89, 287203 (2002).

68. G. Tatara, Y.W. Zhao, M. Munoz, and N. García: Domain wall scattering explains $300 \%$ ballistic magnetoconductance of nanocontacts. Phys. Rev. Lett. 83, 2030 (1999).

69. BiC, SunC, and XuM: Electrical control of metallic heavy-metal-ferromagnet interfacial states. Phys. Rev. Appl. 8, 034003 (2017). 